\title{
Ranking of actions to reduce risks in QMS of construction enterprises
}

\author{
Nikolay Ivanov ${ }^{1, *}$ \\ ${ }^{1}$ Moscow State University of Civil Engineering; 26, Yaroslavskoe sh. 26, 129337 Moscow, Russia
}

\begin{abstract}
In conditions of economic crisis, businesses of construction industry lack the resources to eliminate real risks of non-conformities due differences between construction products and consumers' expectations. In this situation when classic models based on the CAPA become ineffective, the management of non-conformities requires some new acceptable, efficient, and low-cost approaches. The paper describes the original ranking algorithm for measures aimed at reducing the risks in business environment with financing constraints.
\end{abstract}

\section{Introduction}

In the history of any company comes a time when in front of its leadership raises the question about what to do to improve company's performance? This question is relevant as to a successful enterprise, which has quite a large market share, as well as for a company on the verge of survival. ISO 9001:2015 answer the question this way: the company is more successful when its management system and its products meet the requirements of all stakeholders. The process approach, which applied in the Standard and includes the cycle PDCA ("Plan - Do - Check - Act") and risk-based thinking, allows the organization to plan its processes and their interactions. Implementation of a PDCA cycle allows the organization to ensure its processes with the necessary resources and manage them as well as identify and implement opportunities for improvement. Risk-oriented thinking enables an organization to identify the factors that may lead to a deviation from the planned results and to apply preventive controls to minimize negative impacts and maximize the use of emerging opportunities.

As a rule, the aforementioned deviations with negative consequences are called nonconformities. An organization is required to fix detected inconsistencies, identify their causes, plan and implement corrective actions to eliminate the causes of non-conformities.

Any non-conformity appeared during construction of buildings or structures leads to a non-conforming product. Additional investment of time and resources of the organization are the result of processing or disposal of such products. Therefore, activities related to the elimination of nonconforming products (corrections) and with the elimination of the causes of non-conformities (corrective actions) should be considered as sources of additional costs. In some cases, the additional costs of the elimination of nonconforming products may be $28-33 \%$ of the cost of construction. Any construction organization will seek to minimize

* Corresponding author: IvanovNA@mgsu.ru 
these costs. A design of effective corrective action plan can be considered as one of the ways to reduce the additional costs. Consider a plan as an effective plan when the cost of its implementation is significantly lower than potential cost of removal of nonconforming products due non-conformities, which were not removed.

Ideally, any construction enterprise must address the causes of all identified discrepancies, which lead to the emergence of nonconforming products. However, it is not a case in everyday practice. As previously mentioned, the problem lies in the lack of funds for implementation of all necessary corrective actions. Analysis of practice used by building organizations of Russia for the management of non-conformities revealed that most often implemented two options. The first variant is the choice of the least expensive measures from the list of corrective actions. Second variant is the choice of measures aimed at eliminating the causes of the most resonant non-conformities that cannot be ignored. In both cases, the efficiency of the funds used on the corrective actions is not defined and is not considered.

Questions of data collection and data processing related to non-conformities and the reasons for their occurrence are widely enough considered in the scientific literature $[1,2]$. These issues are reflected in a number of previous articles of the author [3, 4]. However, in the scientific literature, problems of formation of corrective action plan are not considered. Separate studies provide only general information about the role of this plan as part of CAPA. Thus, the study aimed at the development of practical recommendations on the formation of the corrective action plan seems very important. In papers published during 2014-2016 [5,6] the author took the steps to formalize the problem. In particular, in [5] describes the algorithm, which selects the primary non-conformity based on priority rank of corrective actions associated with that the non-conformity. Additionally, [5] discusses the algorithm for creating a set of corrective actions to address the root causes of nonconformity. In [6] the author carried out the original ranking algorithm for corrective actions included in the action plan, which aimed at addressing the identified nonconformities and their causes.

This article is another attempt to formulate a methodical approach, which is based on an effective governance mechanism of non-conformities, detected in the process of QMS.

\section{Description of the problem}

\subsection{Verbal description}

Let there be a set of non-conformities, each of which contains a list of reasons that led to the emergence of non-conformity. Let as well, for each of the identified non-conformities there are fixed costs, which would be used on elimination of nonconforming products.

Assume that frequencies of occurrence of different non-conformities were identified. Furthermore, so called chains of non-conformities, which helped to define root causes for all non-conformities, were recorded. Assume also that an expert defined a set of corrective actions to address each of the causes of each of non-conformity. Additionally, the expert identified potential costs associated with the implementation of each of the corrective actions.

It is necessary to determine a procedure to eliminate the causes of non-conformities. The procedure should reduce the risk of occurrence of non-conformities and minimize the costs of the removal of potential nonconforming products.

For simplicity, consider the task in terms that are not directly related to the QMS. Imagine that new completely clean carpet was laid out in receptionist head-manager of the organization. Eventually, the carpet will get all sorts of stains, different sizes and for 
different reasons. It would be nice to take the carpet to the cleaners every night; but, the organization does not have the time or resources. Instead the organization hired a maid, which has the task of keeping the carpet clean. When a stain on the carpet is found, the maid has to document the type of stain and the cause of its occurrence. Then she/he has to clean the carpet with special cleaning products and try to remove the spot entirely to the best of her/his effort. The worst result is that she/he reduces its area and/or the degree of damage. Acquisition costs of cleaning products are included in the enterprise's product cost, thus lowering its competitiveness. The organization made a decision to create a plan of measures on elimination of the known causes of spots in order to prevent re-staining and thus to reduce non-productive expenditures. Note, that the amount of money that can be spent on the elimination of known causes of spots is limited.

We assume that each detected non-conformity $j$, which is caused by the reason $i$, leaves the "spot" of a certain type. The cost of such "spot" removal will be considered equal to the value of the cost of removal of non-conforming products associated with the identified nonconformity $-\mathrm{z}_{\mathrm{np}}^{\mathrm{ji}}$. The graphical representation of the term "spot" is illustrated in the form of a rectangle with sides, equal to 1 and $\mathrm{z}_{\mathrm{np}}^{\mathrm{ji}}$ (Fig. 1a). If any nonconformity $j$, due to the reason $i$, is detected repeatedly, the total area of "spots" of one type ("area of contamination") will be equal to the sum of the areas of elementary rectangles (Fig. 1b) or the area of a rectangle with sides $N_{i j}$ and $\bar{z}_{n p}^{j i}$ (Fig. 1c). Value $N_{i j}$ is the number of times when non-compliance $j$ due to reason $i$ happened. Value $\bar{z}_{n p}^{j i}$ is the average cost of the nonconforming products elimination, which is calculated according to the formula (1):

$$
\bar{Z}_{\mathrm{H \Pi}}^{j i}=\sum_{k=1}^{N i j} z_{n p}^{j i k} / N i j
$$

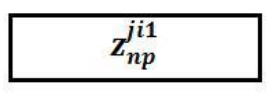

a)
1
2
3

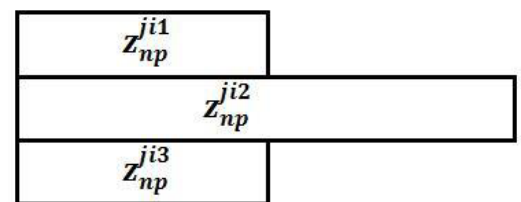

b)

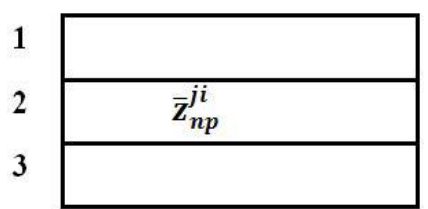

c)

Fig. 1. The cost of removal of nonconforming products ("area of contamination") which is a result of non-conformity $j$ due to reason $i$; a) for a single detection of non-conformity; b) with repeated detection of non-conformity; c) model "average cost".

If there are a few causes of non-conformity $j$, the general "area of contamination" can be defined by the formula for this discrepancy (2):

$$
Z_{n p}^{j}=\sum_{i=1}^{M} \sum_{k=1}^{N i j} z_{n p}^{j i k}
$$

$M$ is the number of identified causes of non-conformity $j$ (the number of "spots" types).

Then the overall cost of the removal of "spots" (full carpet cleaning) $\mathbf{Z}$ can be determined by formula (3):

$$
\mathbf{Z}=\sum_{j=1}^{L} \sum_{i=1}^{M} \sum_{k=1}^{N i j} z_{n p}^{j i k}
$$

$L$ is the number of identified inconsistencies.

The cost of removal of any $i$-th cause of the $j$-th non-conformity is determined by the cost of corrective actions to eliminate this $i$-th cause. Let us call them as $f^{i j}$. 
The cost of removal of any non-conformity is determined by the cost of corrective actions to eliminate all causes of the non-conformity. Let us call these costs as a $F_{c a}^{j}$. The volume of these costs can be determined by formula (4):

$$
F_{c a}^{j}=\sum_{i=1}^{M} f^{i j}
$$

\subsection{Formal description}

We need to create a set of corrective measures, for which the conditions (5) - (8) can be satisfied.

$$
\sum_{j=1}^{L} Z_{n p}^{* j} \rightarrow \min
$$

with constraints

$$
\begin{gathered}
\mathrm{L}^{*}<\mathrm{L} \\
\sum_{j=1}^{L} \sum_{i=1}^{M} f^{i j} \leq \text { Splan } \\
F_{c a}^{j}<Z_{n p}^{j}
\end{gathered}
$$

$L$ - the number of non-compliances identified in the previous period;

$L^{*}$ - the number of possible non-conformities of the planning period (the number of non-conformities of the past period, the cause of which is not completely eliminated);

$M$ - the number of different reasons that resulted in non-conformity $j$;

$f^{i j}$ - the costs of elimination of the reason i, which led to the emergence of nonconformity $j$;

Splan - the amount of funds allocated for corrective measures following the results of the past period;

$Z_{n p}^{j}$ - the costs of removal of nonconforming products related to the identified nonconformity $j$;

$Z_{n p}^{* j}$ - the potential costs of the removal of non-conforming products associated with the re-emergence of non-conformity $j$;

\section{Algorithm of designing an effective corrective action plan}

The original algorithm of designing an effective corrective action plan, "Carpet Cleaning" algorithm, was a result of our research aimed at studying the efficiency of construction companies before and after the introduction of the QMS and the effectiveness of decisions taken to reduce the risk of non-conformities.

The algorithm consists of the following sequence of actions:

1. Identify all types of stains that appeared on the carpet for a certain period of time, and establish the cause.

2. For each of the causes of the spots define an event aimed at their elimination, the costs for the implementation of this action, as well as the total cost of cleaning the carpet from stains that appeared as a result of the considered reason.

3. If the cost of eliminating the causes of any type of stain is greater than the cost of removing all the stains of this type, then limit the actions by only the removal of stains 
("carpet cleaning"); otherwise, in addition to removing the stains, plan to carry out activities to address the causes of spots.

4. Determine the effectiveness for each scheduled event. The efficiency of measures is the ratio of the cost of stains removal, where the particular cause is the reason of stains, to the sum of the all the costs for the elimination of that particular cause. The higher the value of this index, the better the event.

5. Determine the amount of funds needed for the least costly of all the activities included in the plan. Assign the value found for the indicator "Minimum Cost".

6. Sort the planned activities in order of decreasing effectiveness.

7. Set the value of the index "Action Priority" to 1.

8. Choose the most effective measure out of the possible measures that don't have implementation priority.

9. If the costs of the measure are more than the remaining amount of funds allocated for the elimination of the known causes of stains, the measure is removed from the plan and the process proceeds to step 8 .

10. Keep the event in the plan. Set its "Action Priority" index equal to the current value of "Action Priority" index.

11. Reduce the remaining amount of funds allocated for the elimination of the known causes of stains for the cost amount of conducted activities.

12. If the remaining amount of funds allocated for the elimination of the known causes of stains is less than the indicator of "Minimum Cost", go to step 14.

13. Increase the value of the "Action Priority" indicator by 1 and go to step 8.

14. All activities with no sequence of their execution are excluded from the plan.

\section{Summary and conclusions}

Each company has own way of treating risk, and it's the task of risk-team of company to determine the best way to interpret risk levels [7]. Once this is done, risk-team needs to take action on the risks management. They can to make use known quality management processes such as corrective and preventive actions (CAPA) to address the risks. Reviewed above algorithm can be considered as one of the possible ways of solving the problem of minimizing the risks. The advantages of the algorithm are simplicity and scalability. The algorithm can be modified by changing the level of details of the taken decisions.

\section{References}

1. M. Donauer, P. Peças, A. Azevedo, Robot. and Comp.-Integr. Manuf. 36, 84-92 (2015)

2. D. Okes, Root Cause Analysis: The Core of Problem Solving and Corrective Action (ASQ Quality Press, 2009).

3. N. Ivanov, Mod. App. Sc. 10(1), 47-51 (2016)

4. N.A Ivanov, Int. J. Appl. En. Res. 10(21), 42415-42418 (2015)

5. N. Ivanov, J. of Ec. and Entrepreneurship 11-2, 621-626 (2014)

6. N. Ivanov, Proc. Eng. 153, 228-231 (2016)

7. T. Lozier, Quality Digest Magazine, Information on http://www.qualitydigest.com/inside/risk-management-column/030216-what-riskbased-thinking.html 